Ann. Biol. anim. Bioch. Biophys., 1978, 18 (1), 105-117.

\title{
Etude ultrastructurale, chez un poisson téléostéen l'anguille (Anguilla anguilla L.), des processus de minéralisation dans les cas d'une ossification périchondrale de l'arc branchial et d'une apposition secondaire dans l'os vertébral.
}

\author{
par Evelyne LOPEZ, C. A. BAUD, G. BOIVIN, Francine LALLIER \\ Laboratoire de Physiologie générale ef comparée, Muséum national \\ d'Histoire Naturelle. Laboratoire d'Endocrinologie comparée associé \\ au CNRS, 7, rue Cuvier 75005 Paris, France. \\ Instifut de Morphologie, Ecole de Médecine, Genève 1211 4, Suisse.
}

Summary. Ultrastructural study of the mineralization process during perichondral ossification of the gill arch and secondary accretion in the vertebral bone of the eel (Anguilla anguilla $L$.), a teleost fish.

The ultrastructural aspects of two types of mineralization (initial and secondary) were studied in a teleost fish.

1) During perichondral gill arch mineralization, typical active osteoblasts were seen. We observed intracytoplasmic vesicles close to the cytoplasmic membrane. There were two phases of activity in active osteoblasts ; in the first stage, intramitochondrial electron-dense particles and clear « matrix vesicles » from the cell were seen close to the cytoplasmic membrane or further away in the matrix. In the second stage, there were no intramitochondrial particles, matrix vesicles were more electron-dense and they appeared to be nucleation sites for mineral deposit (needles) ; mineral substance then accumulated mostly at the matrix vesicle surface, forming round calcific areas.

2) In secondary osteoblastic accretion, osteoblastic cytoplasmic organelles did not differ from those found in cartilage osteoblasts. We also observed an accumulation of intramitochondrial particles before mineral deposition. During calcification, matrix mineral needles appeared on scattered electron-dense « spherules 》. These structures were not well delimiłed by a distinct membrane. Mineral deposits increased on these spherules from the cell to the mineralized bone matrix.

Many exocytosis vacuoles usually surrounded the osteogenic cells, thus indicating considerable exchange with the adjacent matrix. We conclude that the sequence of mineralization and distribution of mineral deposits in the secondary mineralization of the eel vertebral bone is similar to early mineralization processes in mammals.

\section{Introduction.}

Les processus par lesquels la matrice organique des tissus squelettiques des Mammifères se calcifie sont encore, en dépit de très nombreux travaux récents, l'objef de controverses tout au moins en ce qui concerne les phases tout à fait initiales de la calcification. En 1953 Neuman et Neuman considèrent la matrice extracellulaire comme 
le lieu de nucléation des éléments minéraux sans préciser la nature du site de ce dépôt. Glimcher et Krane (1968) pensent qu'il s'agit du collagène, c'est la théorie «épitaxique » de la calcification ; d'autres proposent les mucopolysaccharides et les mucoprotéines (qui voisinent avec le collagène) ou bien les lipides ef les phospholipides (Boivin, 1975). Le premier, Pautard $(1965,1966)$ émet l'hypothèse d'une participation cellulaire dans les phénomènes de minéralisation. Puis Anderson (1967) ef Bonucci (1967) décrivent respectivement des vésicules et des globules membranés, extracellulaires et osmiophiles, comme étant préférentiellement localisés dans la matrice intercellulaire cartilagineuse ; ils les identifient comme étanł le site du premier dépôt minéral. Ensuite des structures membranées analogues sont observées dans la matrice en cours de calcification de la dentine et de différents tissus osseux mais exclusivement dans le cas de minéralisations primaires (Anderson, 1976). Bernard ef Pease (1969) supposent que les formations membranées observées proviennent de bourgeons émis par les ostéoblastes. Une grande variété de noms a été attribuée à ces structures, ils ont été répertoriés par Anderson (1976), nous les appellerons au cours de l'exposé de nos résultats «structures vésiculaires » (Boivin, 1975) lorsqu'une membrane limitante est décelable et «sphérules opaques aux électrons 》 lorsque cette membrane n'est pas nettement visible. Les structures vésiculaires décrites par Anderson (1969) ef Ascenzi ef Bonucci (1970) sont limitées par une membrane en trois strates renfermant une substance amorphe et osmiophile. Les controverses soulevées par la découverte de telles structures concernant en particulier : leur mode de formation, la nature de leur contenu, le processus d'amorçage de la précipitation de minéral, tirent en grande partie leurs origines du fait que les résultats rapportés et souvent comparés sont oblenus sur des tissus ou des types d'os très différents (Ascenzi et Bonucci, 1970).

Des éclaircissements précieux sur les aspects ultrastructuraux des phénomènes de minéralisation et leurs différentes étapes ont été apportés par l'étude de calcinoses cutanées induites expérimentalement par calciphylaxie locale (Baud et Dupont, 1966 ; Baud et Badonnel, 1967, 1970 ; Gabbiani ef al., 1969). Les phases tout à fait initiales de la calcification cutanée mettant en évidence l'apparition, avant tout dépôt minéral d'apatite dans la matrice collagène, de granules denses intramitochondriaux, ont été montrées par Matthews et al. (1971) et Boivin (1975) ; ce dernier identifie ces granules intramitochondriaux comme étant du phosphate de calcium, il met en évidence la présence de structures vésiculaires typiques à l'endroit où les tissus cutanés se calcifient et étudie les toutes premières précipitations de minéral qu'il montre être de nature apatitique (Boivin et Tochon-Danguy, 1976).

En ce qui concerne certains Vertébrés inférieurs, tels les Poissons téléostéens, de nombreuses structures opaques aux electrons et contenant des cristaux de substance minérale ont été signalées par plusieurs auteurs, dans les écailles, au cours de phénomènes d'apposition ostéoblastique (Maekawa and Yamada, 1970 ; Yamada, 1971 ; Kobayashi ef al., 1972) ; Schönbörner (publication en préparation) y observe des structures vésiculaires et met en évidence leur origine cellulaire. II n'existe pas de données concernant les aspects ultrastructuraux des phénomènes de calcification de l'os des Poissons. Dans ce travail nous étudions les processus de minéralisation chez l'Anguille, d'une part au cours d'une ossification périchondrale, d'autre part lors d'une apposition ostéoblastique due au remaniement osseux. Nous établissons ainsi une comparaison de la séquence de minéralisation et de la répartition du minéral entre les 
processus primaire et secondaire ; jusqu'ici, chez les Mammifères, une calcification partant de la cellule ostéogénique n'a pu être décelée que lors d'un dépôt minéral initial (Bernard, 1969 ; Ascenzi et Bonucci, 1970 ; Bonucci, 1971 ; Anderson et Reynolds, 1973 ; Frank et Stever, 1977).

\section{Matériel ef méthodes.}

Des échaniillons d'arc branchial et d'os vertébral ont été prélevés chez des Anguilles femelles argentées d'un poids $\simeq 200 \mathrm{~g}$ provenant de Peronne (Somme) maintenues au laboratoire depuis deux mois el des fragments d'os vertébral sur des Anguilles femelles argentées pesant environ $300 \mathrm{~g}$. Une autre série de prélèvements vertébraux ont été pratiqués sur des Anguilles argentées (poids $\simeq 300 \mathrm{~g}$ ), ayant subi les unes l'ablation des corpuscules de Stannius (glandes situées sur le rein des Poissons Holostéens et Téléostéens) suivant le protocole mis au point par Leloup-Hatey (1964), les autres le même protocole opératoire, les glandes étant laissées en place.

Prélèvements. Les fragments de tissu ont été prélevés sur les animaux vivants après narcose au MS 222 Sandoz.

\section{Fixations.}

1. - L'arc branchial a été fixé par perfusion du liquide fixateur pendant $10 \mathrm{mn}$ selon une méthode mise au point au laboratoire par Milet ef Martelly-Bagot (publication en préparation), puis par immersion selon un temps différent suivant la nature du fixateur. Deux liquides fixateurs ont été employés :

a) le tétraoxyde d'osmium à 1 p. 100 dans une solution de sucrose $0,25 \mathrm{M}$ additionnée de 7,3 p. 100 de polyvinylpyrrolidon ( $\mathrm{pH}=7,4)$. Temps d'immersion après perfusion : $45 \mathrm{mn}$ à $\mathrm{O}{ }^{\circ} \mathrm{C}$ puis lavage dans une solution de sucrose $0,25 \mathrm{M}(\mathrm{pH}=7,4)$;

b) le glutaraldéhyde à 5 p. 100 tamponné avec du cacodylate de sodium $0,1 \mathrm{M}$ $(\mathrm{pH}=7,2)$. Temps d'immersion après perfusion : $1 \mathrm{~h}$ à $4{ }^{\circ} \mathrm{C}$ puis lavage dans une solution de saccharose $0,2 \mathrm{M}$ dans du tampon cacodylate de sodium $0,1 \mathrm{M}(\mathrm{pH}=7,2)$ suivi d'une post fixation à l'osmium selon le protocole décrit ci-dessous (2).

2. - Les fragments d'os vertébral ont été fixés immédiatement après prélèvement pendant $2 \mathrm{~h}$ à $4{ }^{\circ} \mathrm{C}$ dans du glutaraldéhyde à $5 \mathrm{p}$. 100 tamponné avec du cacodylate de sodium $0,1 \mathrm{M}(\mathrm{pH}=7,2)$ puis lavés dans une solution de saccharose $0,2 \mathrm{M}$ dans du tampon cacodylate de sodium $0,1 \mathrm{M}(\mathrm{pH}=7,2)$ et postfixés $2 \mathrm{~h}$ à $4{ }^{\circ} \mathrm{C}$ dans du tétraoxyde d'osmium à 1 p. 100 dans du tampon cacodylate de sodium 0,1 M (Sabatini ef al., 1963).

Inclusions. Les échantillons fixés selon ces différents protocoles ont été déshydratés puis inclus dans l'épon B.

Coupes ef colorations. Les coupes ont été réalisées au microtome Porter Blum 1 avec un couteau de diamant. Toutes les coupes ont été colorées, les unes à l'acétate d'uranyle citraje de $\mathrm{Pb}$, les autres au citrate de $\mathrm{Pb}$ seul (Vénable et Coggeshall, 1965) de façon à éviter certaines déminéralisations (Baud, résultats inédits).

Les micrographies électroniques ont été réalisées à l'aide du microscope Hitachi HU 12. 


\section{Résultats.}

\section{Ossification primaire de l'arc branchial.}

L'ossification de l'arc branchial des Poissons Téléostéens est du type périchondral ; elle se fait au contact d'un nodule cartilagineux constitué de cartilage hyalin, ce cartilage peut persister ou disparaître (Blanc, 1953). Les aspects ultrastructuraux de cette ossification périchondrale seront décrits dans un mémoire ultérieur. Nous n'envisageons ici que la participation cellulaire au processus de minéralisation. Au pourtour du nodule cartilagineux on observe des ostéoblastes typiques (fig. 1, 2, 4) présentant un réticulum endoplasmique granulaire (ergastoplasme), un appareil de Golgi important, de petites mitochondries et de nombreuses vésicules situées près de la membrane cytoplasmique et qui sont probablement d'origine endoplasmique. On distingue des microfilaments, quelques microtubules et parfois de gros lysosomes. On peut, en ce qui concerne ces ostéoblastes, distinguer deux stades :

- l'un est marqué par la présence de mitochondries riches en petits granules denses de 220 à $350 \AA$ de diamètre (fig. 5) et de nombreuses vésicules intracytoplasmiques situées au pourtour de la cellule près de la membrane (fig. $1,2,4)$. Ces ostéoblastes sont séparés de la substance fondamentale du cartilage par une matrice lâche comportant notamment des fibrilles de collagène entrecroisées à striation transversale non apparente ; on observe à l'extérieur de la cellule, soit près de la membrane cytoplasmique, soił plus éloignées des structures vésiculaires membranées situées entre les fibrilles de collagène et dont le contenu est peu opaque aux électrons, leur taille varie entre 350 et $1200 \AA$ (fig. 2, 3). Une telle variabilité de taille des vésicules comprise entre 500 et $1000 \AA$ a été mentionnée par Slavkin et al. (1976) dans le cartilage.

\section{PLANCHE ।}

FIG. 1. - Ostéoblaste observé dans l'arc branchial d'une Anguille, près du front de minéralisation. Mitochondries ( $m$ ) avec granules opaques aux électrons. Vésicules intracytoplasmiques (vi). Microfilaments (mf). Ergastoplasme (er). $\times 22500$.

FIG. 2. - Partie d'ostéoblaste observée dons l'arc branchial d'une Anguille, près du front de calcification. Mitochondries avec petits granules opaques $(\mathrm{m})$. Microfilaments $(\mathrm{mf})$. Vésicules intracytoplasmiques (vi). Structures vésiculaires membranées peu opaques aux électrons $(\rightarrow)$ situées dans la matrice collagène $(\mathrm{mc})$ à proximiłé de la cellule ou plus éloignées. $\times 18000$.

FIG. 3. - Structures vésiculaires membranées, opaques aux électrons $(\rightarrow)$, observées dans la matrice collagène entre la cellule (fig. 2) ef le front de calcification. $\times 18000$.

FIG. 4. - Partie d'ostéoblaste (même situation que les ostéoblastes fig. 1, 2). Miłochondrie avec granules opaques aux électrons (m). Appareil de Golgi $(\mathrm{g})$. Vésicules intracytoplasmiques en contact étroit avec la membrane $(\rightarrow)$. $\times 40500$.

FIG. 5, - Mifochondrie observée dans un prolongement ostéoblastique situé au contact du noyau cartilagineux d'un arc branchial. Granules opaques aux électrons $(\rightarrow)$ situés près des crêtes mitochondriales $\times 63000$. 


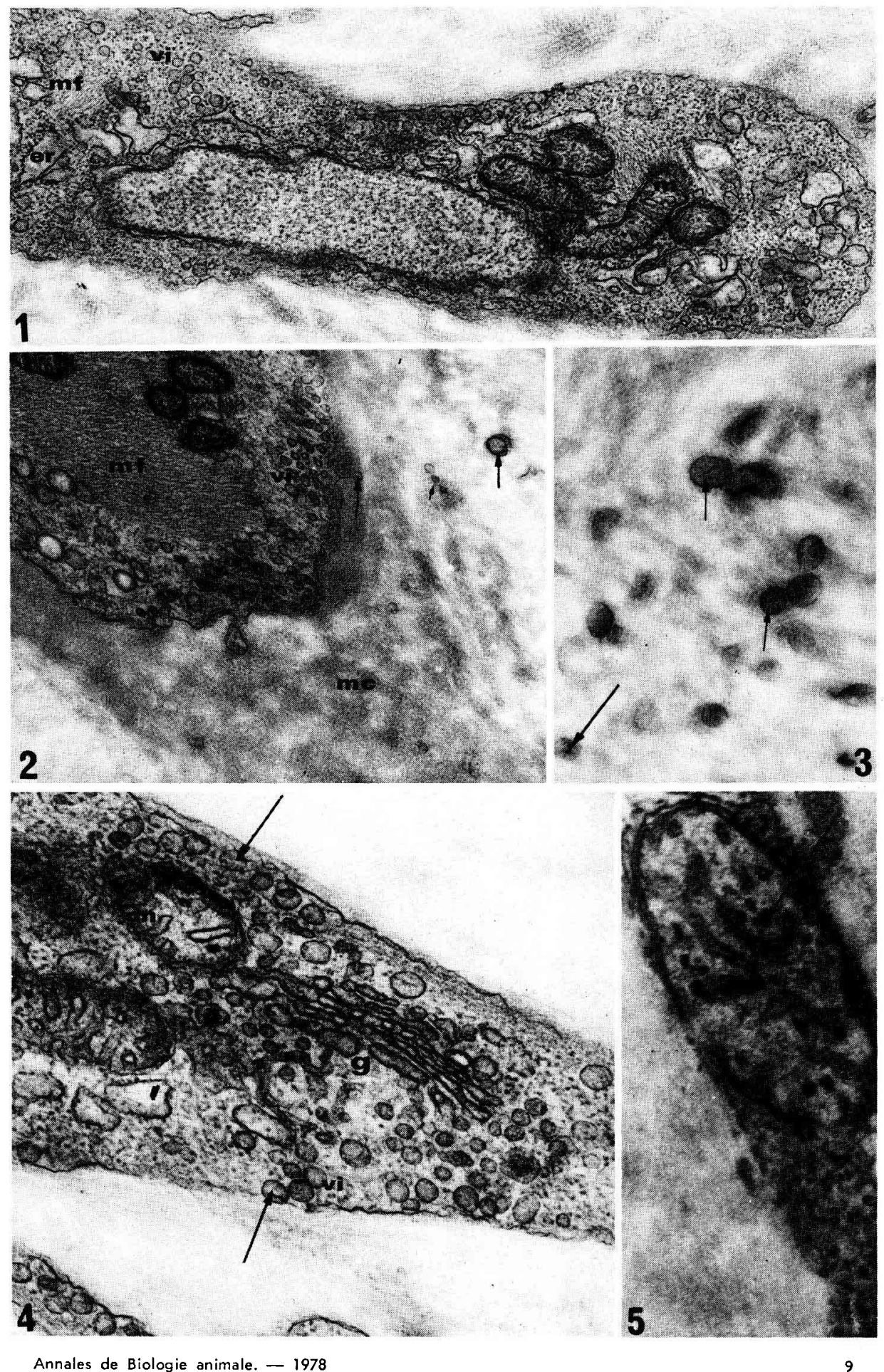


- l'autre caractérise les ostéoblastes situés près du front de calcification ; leurs mitochondries sont pratiquement dépourvues de granules denses et les vésicules intracytoplasmiques sont plus rares. Dans la matrice collagène on peut voir que les structures vésiculaires sont plus denses et on y observe les premières précipitations de minéral (fig. 6). A proximité de la cellule ostéogénique sur (ou dans) chaque vésicule on distingue une ou plusieurs aiguilles de substance minérale, puis près de la matrice calcifiée ces aiguilles se groupent en rosettes sur un substrat dense, la structure strictement vésiculaire disparaît (fig. 6, 8). Sur coupe longitudinale d'un faisceau de fibrilles de collagène on aperçoit des rosettes se développant sur le collagène, les aiguilles sont disposées hélicoïdalement autour des fibrilles(fig. 6). Des observations analogues ont été faites par Boivin (1975) dans le cas de calcinoses cutanées expérimentales.

2. Apposition ostéoblastique due au remaniement osseux (minéralisation secondaire).

L'os vertébral des Poissons Téléostéens (os fibrillaire à fines fibres parallèles) est soumis à un important remaniement de surface, les cellules qui y participent ont été décrites lors d'observations faites en microscopie photonique (Lopez, 1973).

L'aspect ultrastructural des ostéoblastes apparaît être comparable dans les grandes lignes à celui décrit dans ces mêmes cellules, chez les Mammifères. En cas d'apposition les ostéoblastes bordent en couche monocellulaire la matrice calcifiée ; ils sont séparés de celle-ci par une région non minéralisée où le collagène à striations transversales bien marquées est apparent (fig. 7). C'est le tissu ostéoïde des Mammifères que pour des raisons déjà exposées (Lopez, 1973) nous appellerons matrice collagène ; c'est cette matrice que les ostéoblastes sécrètent dans une phase initiale. Ce sont de grosses cellules, plus volumineuses que celles observées dans le cartilage branchial, caractérisées par un ergastoplasme extrêmement abondant (fig. 7, 10) et un appareil de Golgi important. Microfilaments, microtubules et gros lysosomes granulaires sont aussi observés dans le cytoplasme. Comme dans le cas de l'ossifica-

\section{PLANCHE ॥}

FIG. 6 et 7. - Comparaison entre deux processus de minéralisation chez l'Anguille, l'un observé dans le cartilage branchial (fig. 6) l'oufre dans l'os vertébral au cours d'une apposition ostéoblastique secondaire (fig. 7). Dans les deux cas on observe : 1) une grande activité des ostéoblastes ergastoplasme (er), vésicules intracytoplasmiques (vi), exocytose (ex). 2) une répartition comparable du dépôt de substance minérale dans la matrice, sur des structures opaques aux électrons $(\rightarrow)$, les amas devenant plus compacts près de la matrice calcifiée $(\Rightarrow) . \quad \times 15000$.

FIG. 6. - Aiguilles disposées hélicoïdalement autour de fibrilles de collagène coupées longitudinalement $(\triangleright)$.

FIG. 8. - Front de minéralisation au contact du cartilage branchial. Aiguilles de substance minérale groupées en rosettes sur un substrat dense, la structure vésiculaire a disparu. Substance fondamentale du cartilage (sf). Matrice collagène $(\mathrm{mc}) . \quad \times 45000$.

FIG. 9. - Fort grossissement de la région délimitée fig. 7. Sphérules opaques aux électrons sur lesquelles se déposent les premières aiguilles de substance minérale $(\rightarrow) . \quad \times 100000$. 


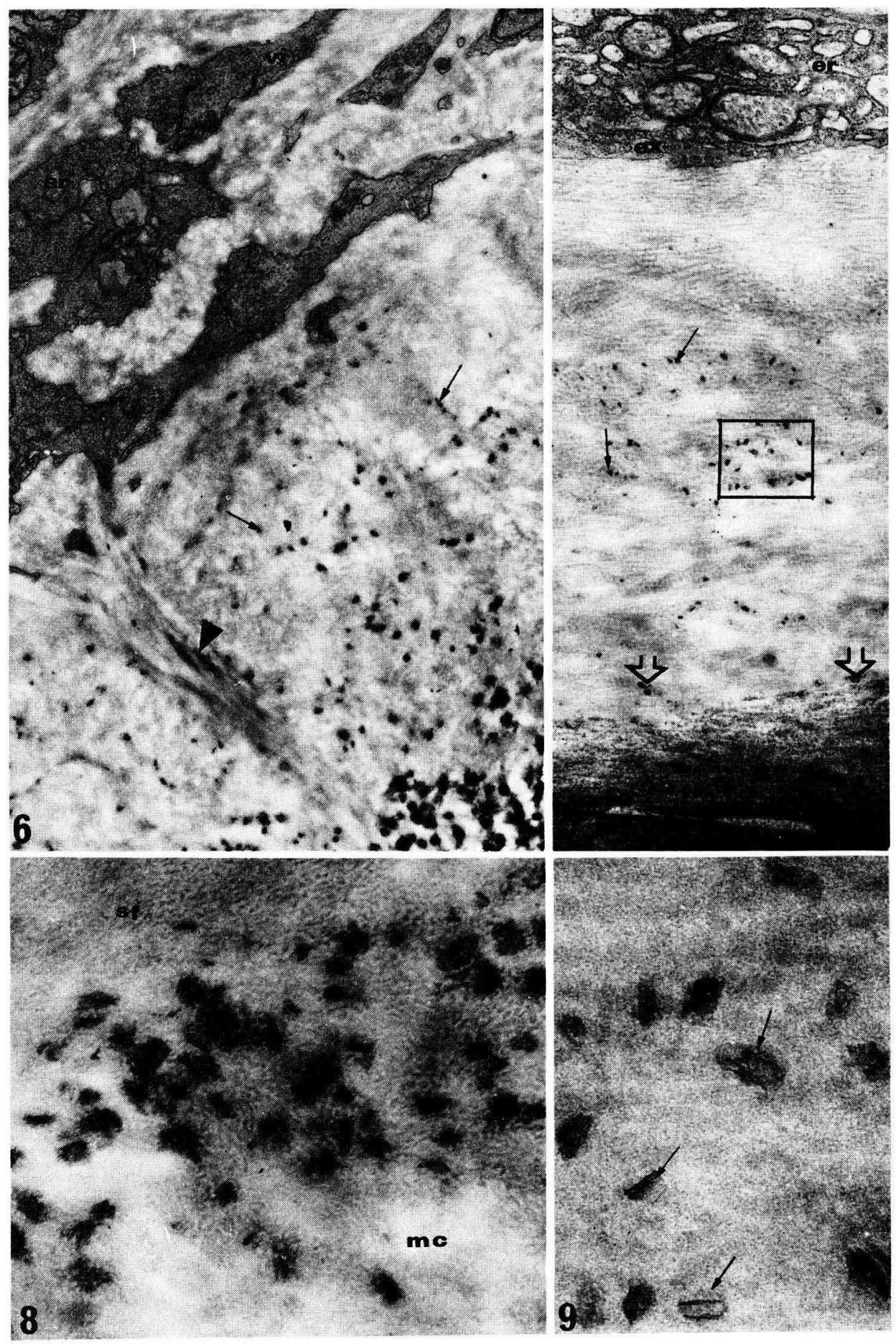


tion périchondrale, certains ostéoblastes, les plus éloignés de la surface osseuse en cours d'apposition ont des mitochondries avec des granules denses (200 à $300 \AA$ ), des vésicules intracytoplasmiques comparables à celles décrites précédemment sont observées au pourtour de la cellule (fig. 7). A l'extérieur de l'ostéoblaste ef à son contact ou bien dans l'ensemble de la matrice collagène, des structures membranées non encore chargées de minéral et comparables à celles observées dans le cas de l'arc branchial ne sont pas identifiables avec netteté. Lorsque la phase de minéralisation est amorcée on n'observe plus de granules denses dans les mitochondries des ostéoblastes, mais dans la matrice en cours de calcification apparaissent de nombreuses sphérules opaques aux électrons sans membrane netfement décelable (300 à $900 \AA$ ) (fig. 7, 9). On peut voir près de la cellule, sur ou dans ces sphérules, les premières précipitations minérales sous forme d'aiguilles (fig. $9,13,14$ ), puis les amas deviennent plus compacts près de la matrice déjà minéralisée (fig. 7,12 ). La répartition du dépôt de substance minérale ainsi que sa séquence sont tout à fait comparables à ce que nous avons montré dans le cas du cartilage.

Lors d'une forte stimulation de l'apposition ostéoblastique, après ablation des corpuscules de Stannius (Lopez, 1970) nous avons remarqué que les sphérules denses avec précipitations minérales sont extrêmement nombreuses (fig. 10,11,12) comparées à ce qu'elles sont chez les témoins. Les ostéoblastes sont alors très stimulés, leurs miłochondries sont dépourvues de granules et les vésicules intracytoplasmiques sont pratiquement inexistantes (fig. 10).

Dans les deux processus de minéralisation que nous venons de décrire nous avons pu faire des observations (plus nettement dans le cas du cartilage) permettant de penser que les vésicules ou sphérules initiatrices de la calcification sont émises par extrusion (fig. 2, 4, 16). Notons que les tailles des vésicules intracellulaires sont com-

\section{PLANCHE III}

FIG. 10, 11, 12. - Reconstitution d'une séquence de minéralisation (os vertébral) lors d'une apposition ostéoblastique stimulée. $\times 15000$.

FIG. 10. - Ostéoblaste hyperactif, mitochondries sans granules (m), ergastoplasme (er), lysosomes (ly).

FIG. 11. - Sphérules très abondantes avec précipitation d'aiguilles de substance minérale (comparer arec fig. 7).

FIG. 12. - Matrice calcifiée en cours de minéralisation secondaire.

FIG. 13. - Fort grossissement montrant des dépôts de minéral observés près de la matrice calcifiée (os vertébral). Les bôtonnets sont groupés en faisceaux sur des structures opaques rappelant les sphérules $(\rightarrow) . \times 100000$.

FIG. 14. - Os veriébral. Sphérules situées dans la matrice $(\mathrm{mc})$, près d'un ostéoblaste, avec premiers dépôts de substance minérale $(\rightarrow) . \quad \times 70000$.

FIG. 15. - Arc branchial. Vacuoles d'exocytose situées à la périphérie des ostéoblastes. $\times 18000$.

FIG. 16. - Arc branchial. Mise en évidence au niveau de la membrane cyłoplasmique d'un ostéoblaste de l'extrusion de vésicules $(\rightarrow)$ dans la matrice collagène $(\mathrm{mc}) . \quad \times 18000$. 


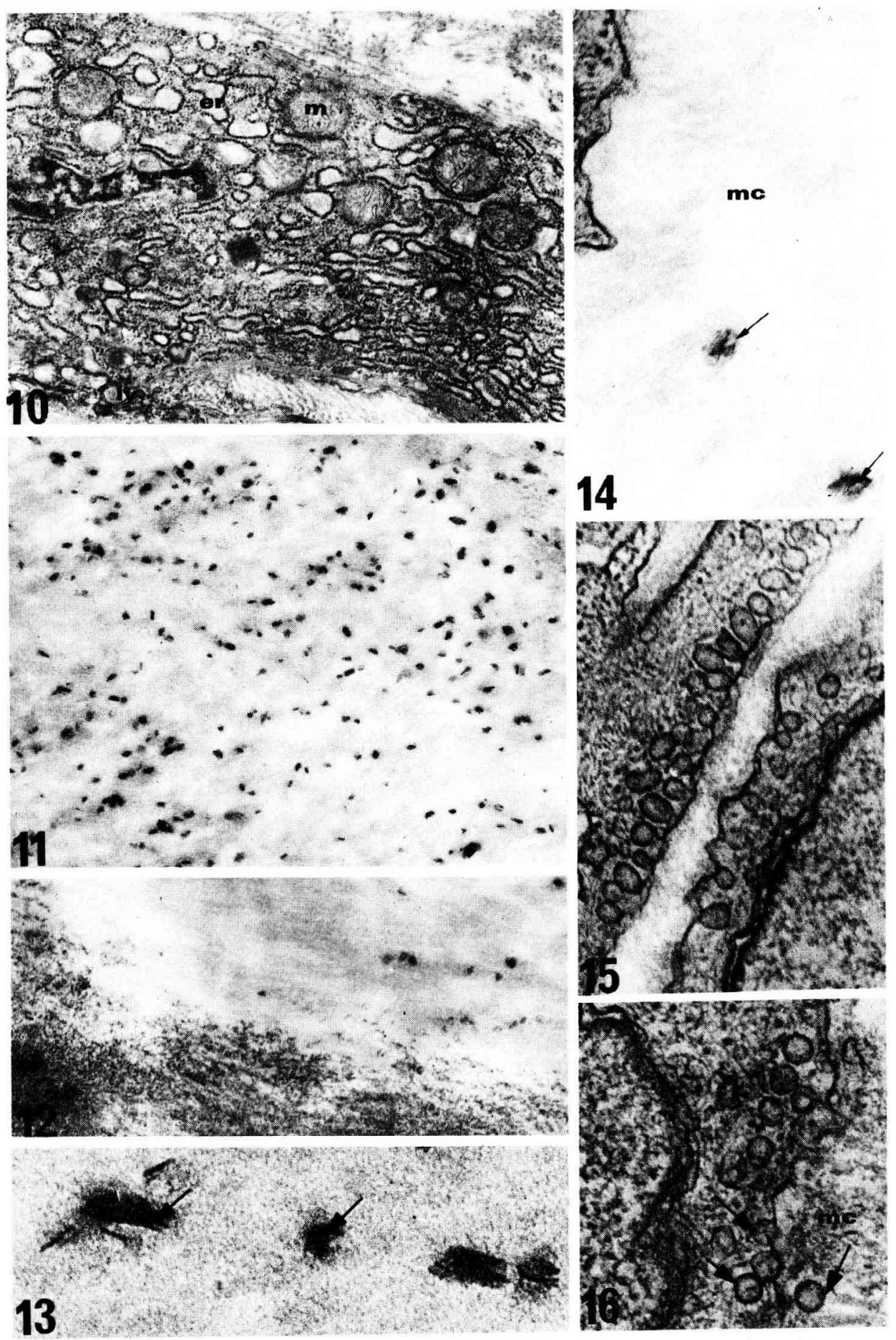


prises entre des limites comparables à celles des vésicules ou sphérules extracellulaires. En outre nous avons observé au niveau de la membrane cytoplasmique des ostéoblastes de très nombreuses vacuoles avec exocytose et émission de matériel ; ces vacuoles sont disposées à la périphérie de la cellule, sans polarité (fig. 7,15$)$.

\section{Discussion.}

Il est clairement démontré aujourd'hui que chez les Mammifères les chondrocytes et les ostéoblastes interviennent directement dans le mécanisme de calcification (Ascenzi et Bonucci, 1970 ; Bonucci, 1971 ; Anderson, 1976) ils pourvoient au transport des ions calcium du sang à la matrice en cours de calcification et produisent, selon des modalités différentes, suivant la nature du tissu, des structures vésiculaires qui induisent la formation du minéral (cf. Conference on matrix vesicle calcification 1976).

Toutefois la présence, chez les Vertébrés supérieurs, de vésicules de calcification d'origine cellulaire n'a été mise en évidence, ainsi que nous l'avons déjà souligné, que dans les minéralisations initiales. Au cours de l'éfude que nous avons faite, nous nous sommes posés plusieurs questions.

1. Y a-t-il similitude entre les deux processus de minéralisation observés; ceux-ci sont-ils comparables à ce qui a été décrit chez les Mammifères? Qu'il s'agisse de la calcification primaire ou de l'apposition secondaire les premiers dépôts du minéral se font en allant de la cellule ostéogénique vers le front de minéralisation. Ces dépôts minéraux apparaissent dans le premier processus sur des structures vésiculaires typiques dont on a pu observer neftement la membrane, dans le second sur des sphérules le plus souvent aux contours flous mais cependant nettement différenciées du reste de la matrice. Les tailles de ces deux types de formations sont à peu près comparables car très variables dans l'un et l'autre cas, elles peuvent être plus grosses (1 000 à $1200 \AA$ ) dans le cartilage. Nous pouvons supposer que l'absence de membrane nette autour des sphérules est due au fait qu'il ne s'agit pas du même mode de fixation. Nous avons en effet remarqué que l'osmium, en perfusion, fixe d'une façon excellente toutes les membranes dans les tissus des Poissons, contrairement au glutaraldéhyde. Ce point est en cours d'étude. Toutefois il est généralement relaté dans l'ensemble des travaux récents sur les sites de calcification que dès l'apparition du dépôt minéral, la membrane de la vésicule devient moins distincie.

La séquence de minéralisation ainsi que la répartition du dépôt minéral sont tout à fait comparables dans les deux sortes de processus envisagés et ne diffèrent pas de ce qui est décrit chez les Mammifères lors de calcifications initiales au niveau des tissus conjonctifs (Boivin, 1975 ; Conference on matrix vesicle calcification, 1976) ni de ce qui a été montré chez certains téléostéens lors de minéralisation des écailles (Maekawa and Yamada, 1970 ; Yamada, 1971 ; Kobayashi ef al., 1972 ; Schönbörner, publication en préparation).

2. Quel est le mode de formation des structures vésiculaires et le mécanisme de leur évolution ? Les opinions sont très divergentes à ce sujet, Rabinovitch ef Anderson (1976) proposent quatre modes : émission de bourgeons cellulaires, extrusion de vésicules préformées, désintégration cellulaire, sécrétion de sous-unités ensuite assemblées 
dans la matrice collagène. Les images que nous avons obtenues nous permettent d'opter, tout au moins en ce qui concerne notre matériel, pour le second mode. Ces observations sont faites plus nettement dans le cas du cartilage, cette phase semble plus difficile à saisir lors de l'apposition ostéoblastique. En effet il s'agit d'un phénomène dynamique que l'on met en évidence par une méthode morphologique. II est remarqué que lors d'une stimulation expérimentale de l'apposition le nombre des sphérules de calcification est augmenté dans de fortes proportions ; leur sécrétion est-elle sous contrôle hormonal ? De nombreuses vésicules ou sphérules, assez transparentes aux électrons, et souvent situées près de la cellule ostéogénique laissent à penser qu'elles ne contiennent pas tout leur stock minéral lors de leur formation. Le contenu de ces premières formations serait en grande partie organique ef les ions minéraux se concentreraient ensuite par l'intermédiaire de mécanismes de passages préférentiels. Nous avons signalé la présence de nombreuses vacuoles bordant la membrane cellulaire et débouchant par exocytose dans la matrice. Cette observation traduit la présence d'échanges très importants entre la cellule et la matrice en cours de calcification qui étayent l'hypothèse émise sur la charge progressive des sites de calcification, le matériel étant fourni à la matrice collagène par la cellule. Des vacuoles similaires, dont l'activité est liée aux mitochondries, ont été décrites par Somlyo et al. (1974) dans le muscle lisse ; elles sont en relation avec des transferts de cations notamment du calcium.

3. Quel est le rôle des mitochondries? Il est bien connu actuellement, chez les Mammifères, que la présence de granules denses, intramitochondriaux (Gonzales et Karnovsky, 1961 ; Baud, 1962 ; Matthews et al., 1971 ; Boivin, 1975) et identifiés comme étant de nature minérale (Boivin, 1975) représente une accumulation de phosphate de calcium dans la cellule et dans le cas présent un słockage en vue d'une calcification. La mise en évidence de la présence de ces granules, dans les deux types de minéralisation observées chez l'Anguille, puis leur disparition lors des premiers dépôts minéraux, montrent un processus comparable à ce qui est décrit chez les Vertébrés supérieurs.

\section{Conclusions.}

Chez l'Anguille, la séquence de minéralisation et la répartition du minéral dans le cas d'une apposition secondaire sont comparables à celles d'une minéralisation primaire. Le dépôt préférentiel d'aiguilles minérales sur des sphérules opaques aux électrons, que nous avons observé dans les deux cas, n'a pu être jusqu'ici démontré chez les Vertébrés supérieurs lorsqu'il s'agit d'une apposition sur une surface déjà minéralisée (Frank et Steuer, 1977). On peut penser que cette différence est due à une variabilité de vitesse du phénomène, celui-ci étant si rapide chez les Mammifères, qu'il ne peut être saisi, ou à une différence phylogénétique en ce qui concerne l'activité de l'ostéoblaste. Les Poissons sont un matériel de choix pour l'étude de ces sites de calcification et en particulier d'une éventuelle régulation hormonale du phénomène.

Réunion Groupe Développement INRA/Productions animales Montpellier, 17-18 mai 1977. 
Remerciements. - Nous remercions vivement Francis Fernandes pour l'illustration. Ce travail a été effectué grâce au contrat ATP CNRS Tissus calcifiés nº 1305.

\section{Références}

ANDERSON H. C., 1967. Electron microscopic studies of induced cartilage development and calcification. J. Cell Biol., 35, 81-101.

ANDERSON H. C., 1969. Vesicles associated with calcification in the matrix of epiphyseal cartilage. J. Cell Biol., 41, 59-72.

ANDERSON H. C., 1976. Matrix vesicle calcification. Fed. Proc., 35, 105-108.

ANDERSON H. C., REYNOLDS J. J., 1973. Pyrophosphate stimulation of calcium uptake into cultured embryonic bones. Fine structure of matrix vesicles and their role in calcification. Dev. Biol., 34, 211-227.

ASCENZI A., BONUCCI E., 1970. Etude comparée au microscope électronique des phases initiales de la calcification de l'os ef du cartilage 65-77. In HIOCO D. J., Phosphate et méfabolisme phosphocalcique, Sandoz, Paris.

BAUD C. A., 1962. Morphologie et structure inframicroscopique des ostéocytes. Acta anat., 51, 209225.

BAUD C. A., BADONNEL M. C., 1967. Efude par microscopie et diffraction électroniques de la calcinose cutanée expérimentale. Acta anat., 68, 600-601.

BAUD C. A., BADONNEL M. C., 1970. Electron microscope and electron diffraction study of experimental cutaneous calcinosis. Clin. Orthop., 69, 55-65.

BAUD C. A., DUPONT D. H., 1966. La structure submicroscopique des dépôts de substance minérale dans la calcinose cutanée expérimentale (calciphylaxie). Experientia, 22, 18.

BERNARD G. W., 1969. The ultrastructural interface of bone crystals and organic matrix in woven and lamellar endochondral bone. J. dent. Res., 48, suppl. 5, 781-788.

BERNARD G. W., PEASE D. C., 1969. An electron microscopic study of initial intramembranous osteogenesis. Am. J. Anat., 125, 271-290.

BLANC M., 1953. Contribution à l'étude de l'ostéogenèse chez les poissons téléostéens. Mém. Muséum national d'Histoire naturelle, nouvelle série, sér. A, Zoologie, 7, 1-156.

BOIVIN G., 1975. Etude chez le rat d'une calcinose cutanée induite par calciphylaxie locale. I. Aspects ultrastructuraux. Arch. Anat. micros. Morphol. exp., 64, 183-205.

BOIVIN G., TOCHON-DANGUY H. J., 1976. Etude chez le rat d'une calcinose cutanée induite par calciphylaxie locale. II. Aspects biophysiques de la substance minérale. Ann. Biol. anim. Biochim. Biophys., 16, 869-878.

BONUCCI E., 1967. Fine structure of early cartilage calcification. J. Ultrastruct. Res., 20, 33-50.

BONUCCI E., 1971. The locus of initial calcification in cartilage and bone. Clin. Orthop., 78, 108-139.

CONFERENCE on matrix vesicle calcification, 1976. Fed. Proc., 35, 105-171.

FRANK R. M., STEUER P., 1977. Ultrastructure de la surface ligamentaire de l'os alvéolaire du rat. J. Biol. buccale, 5, 55-75.

GABBIANI G., BADONNEL M. C., BAUD C. A., 1969. Relationship between iron, calcium and phosphate during experimental cutaneous calcinosis. Calcif. Tiss. Res., 4, 224-230.

GLIMCHER M. J., KRANE S. M., 1968. The organization and structure of bone and the mechanism of calcification. In GOULD B. S., RAMACHANDRAN G. N., Treatise on collagen. Vol. 2B, chap. 2, 68-251. Acad. Press.

GONZALES F., KARNOVSKY N. J., 1961. Electron microscopy of osteoclasts in healing fractures of rat bone. J. biophys. biochem. Cytol., 9, 299-316.

KOBAYASHI S., YAMADA J., MAEKAWA K., OUCHI K., 1972. Calcification and nucleation in fish scales. Biomineralisation, 6, 84-90.

LELOUP-HATEY J., 1964. Modifications de l'éçuilibre minéral de l'Anguille consécutives à l'ablation des corpuscules de Stannius. C. R. Soc. Biol., Paris, 158, 711-715.

LOPEZ E., 1970. L'os cellulaire d'un Poisson Téléostéen. Anguilla anguilla L. Il. Action de l'ablation des corpuscules de Stannius. Z. Zellforsch. mikroscop. Anat., 109, 566-572.

LOPEZ E., 1973. Efude morphologique et physiologique de l'os cellulaire des Poissons Téléostéens. Mém. Muséum national d'Histoire naturelle, sér. A, Zool., 80. 
MAEKAWA K., YAMADA J., 1970. Some histochemical and fine structural aspects of growing scales of the rainbow trout. Bull. Fac. Fish. Hokkaido Univ., 21, 70-78.

MATTHEWS J. L., MARTIN J. H., ARSENIS C., EISENSTEIN R., KUETTNER K., 1971. The role of mitochondria in intracellular calcium regulation, 239-252. In NICHOLS G., WASSERMAN R. H. Cellular mechanisms for calcium transfer and homeostasis. Acad. Press, N.-Y., London.

NEUMAN W. F., NEUMAN M., 1953. The nature of the mineral phase of bone. Chem. Rev., 53, 1.45 .

PAUTARD F. G. E., 1965. Calcification in baleen, 347-357. In : RICHELLE L. J., DALLEMAGNE M. J., Colcified tissues. Symp. de Liège, 1964.

PAUTARD F. G. E., 1966. A biomolecular survey of calcification, 108-122. In FLEISCH H., BLACKWOOD H. J. J., OWEN M., Calcified tissues. Symp. de Davos, 1965, 108-122.

RABINOVITCH A. L., ANDERSON H. C., 1976. Biogenesis of matrix vesicles in cartilage growth plates. Fed. Proc., 35, 112-116.

SABATINI D. D., BENSCH K., BARRNETT R. J., 1963. Cytochemistry and electron microscopy. The preservation of cellular ultrastructure and enzymatic activity by aldehyde fixation. J. Cell. Biol., 17, 19-58.

SLAVKIN H. C., CROISSANT R. D., BRINGAS P., MATOSIAN P., WILSON P., MINO W., GUENTHER H., 1976. Matrix vesicle heterogeneity : possible morphogenetic functions for matrix vesicles. Fed. Proc., 35, 127-134.

SOMLYO A. P., SOMLYO A. V., DEVINE C. E., PETERS P. D., HALL T. A., 1974. Electron microscopy and electron probe analysis of mitochondrial cation accumulation in smooth muscle. J. Cell. Biol., 61, 723-742.

VENABLE J. H., COGGESHALL R., 1965. A simplified lead citrate stain for use in electron microscopy. J. Cell. Biol., 25, 407-408.

YAMADA J., 1971. A fine structural aspect of the development of scales in the chum salmon fry. Bull. jop. Soc. Sci. Fish., 37, 18-29. 